\title{
Calidad biológica y bioquímica de la población de Artemia (Anostraca: Artemiidae) localizada en las salinas de Real de Salinas, Calkiní, Campeche, México
}

\author{
Leticia G. Rodríguez-Canché1, Teresita DNJ Maldonado-Montiel² \& Luciano A. Carrillo Navarro ${ }^{3}$ \\ 1 Centro de Estudios de Desarrollo Sustentable y Aprovechamiento de la Vida Silvestre, Universidad Autónoma de \\ Campeche, Av. Agustín Melgar s/n entre calle 20 y Juan de la Barrera, Campeche 24030, Campeche, México; \\ lgrodrig@uacam.mx. Tel: 52 (981) 8119800 ext.62500 \\ 2 Facultad de Ciencias Químico Biológicas, Universidad Autónoma de Campeche, México. Tel: 52 (981) 8119800 ext. 73005 \\ 3 Industrias Pecis, S.A. de C.V. Calle 47 No. 266 x 36 y 38. Col Benito Juárez Norte. Yucatán, México. Tel: 52 (99) \\ 99449645
}

\author{
Recibido 02-IV-2002. Corregido 09-V-2006. Aceptado 21-VII-2006.
}

\begin{abstract}
Biological and biochemical quality of the Artemia (Anostraca: Artemiidae) population from Real de Salinas saltworks, Calkiní, Campeche, Mexico. Cysts of Artemia spp. collected from February 1997 to February 2000 in the Real de Salinas solar saltworks, Campeche, Mexico, were compared with Artemia franciscana (batch number 8131 Microfeast Artemia Cysts, Texas, USA). The variables determined in these two populations were: number of cysts per gram, hatching percentage, hatching efficiency, hatching rate, hatching synchrony and hatching biomass, as well as diameter of the cysts and length of the nauplii (instar I). For Salinas, the average diameters of the encapsulated and decapsulated cysts were $230.5 \pm 4.14$ and $221.8 \pm 3.39 \mu \mathrm{m}$, respectively. The thickness of the cyst shell was $4.35 \pm 0.68 \mu \mathrm{m}$ and the length of the nauplii was $388.11 \pm 4.39 \mu \mathrm{m}$, this last value is among the smallest reported in the literature. For the commercial population of $A$. franciscana, the average diameters of the encapsulated and decapsulated cysts were $230.21 \pm 12.49$ and $216.96 \pm 13.71$, respectively. With respect to the corion thickness and length of the nauplii the values were $6.62 \pm 2.72$ and $424.70 \pm 30.08$, respectively. The protein value of the cysts (47.91\%) and nauplii (50.5\%) of Artemia population from Real de Salinas, are considered adequate to be used as food in aquaculture. The results indicate that the population from Real de Salinas presents positive features for its use in aquaculture in the region. Rev. Biol. Trop. 54 (4): 1283-1293. Epub 2006 Dec. 15.
\end{abstract}

Key words: Brine shrimp, live food, crustacean, nutrition, artemia cysts.

En acuicultura, el contar con un alimento adecuado en tipo y calidad para larvas de diferentes especies de interés comercial es un aspecto de suma importancia, ya que la larva, al terminar de absorber el saco vitelino que le proporciona los requerimientos proteicos para su desarrollo durante las primeras $24 \mathrm{~h}$ de vida, iniciará su alimentación exógena y enfrentará lo que en acuiculura se conoce como el punto crítico de la nutrición. El desarrollo deficiente del sistema digestivo de los estadios larvales, impide la adecuada asimilación del alimento artificial (Holt y Sun 1991), por lo que es vital el abastecimiento de alimento vivo. Entre las dietas vivas utilizadas en larvicultura de peces y crustáceos, los nauplios del crustáceo Artemia spp., constituyen el alimento más ampliamente utilizado a lo ancho del mundo (Bengtson et al. 1991). Contar con lotes de quistes de este organismo, que cumplan los criterios estándares de calidad, representa un insumo de gran valor, el cual puede ser utilizado en cualquier momento.

La presencia de artemia en forma natural en las salinas Real de Salinas, Campeche, México, la importancia de la artemia como alimento en acuicultura y el creciente desarrollo de la actividad en el región, motivaron el presente estudio. Contar con una población 
natural de artemia en el lugar donde se practica la actividad acuícola, implica reducir los costos de producción de los organismos en cultivo en los cuales la artemia constituye un alimento, en alguna etapa de su desarrollo.

El uso de artemia como alimento en acuicultura, depende entre otros factores, de su calidad. Los criterios estándares fundamentales para evaluar la calidad de los quistes para su empleo en larvicultura, básicamente son: porcentaje de eclosión $(\% \mathrm{H})$, eficiencia de eclosión (HE), tasa de eclosión (HR), sincronía (TS) y biomasa de eclosión (HO). Otras evaluaciones para caracterizar a una población de Artemia spp. se obtienen del diámetro de quistes, longitud de los nauplios (instar I) y número de quistes por gramo. Considerando la importancia que tienen estos indicadores en acuicultura, el objetivo de este trabajo se dirigió a evaluar los criterios estándares de calidad de los quistes provenientes de la población de Artemia spp. de Real de Salinas, Calkiní, Campeche, México y compararlos con los obtenidos con un lote comercial de Artemia franciscana (lote No. 8131 Microfeast Artemia Cysts, Texas, USA).

\section{MATERIALES Y MÉTODOS}

Descripción del área de estudio: la región salinera de "Real de Salinas" se encuentra al norte del Estado de Campeche, México, en el municipio de Calkiní, en los paralelos $20^{\circ} 02^{\prime}$ $\mathrm{N}$ y $90^{\circ} 14^{\prime} \mathrm{W}$, a $10 \mathrm{msnm}$. La presencia de Artemia sp. se ha detectado en charcas de marea naturales que se localizan dentro de la región decretada como Zona Especial de Protección de Flora y Fauna Silvestre y Acuática "Los Petenes" (Diario Oficial 1996) en el Estado de Campeche; así como también del área de Refugio Faunístico de la Ría Celestún (Diario oficial 1979) en el Estado de Yucatán. La extensión que abarca la región salinera es de aproximadamente 60 ha entre los dos Estados (Comisión Nacional del Agua 1996).

Colecta, procesamiento y análisis del quiste: en 1997 los muestreos se realizaron cada mes a excepción del mes de agosto, en 1998 el muestreo se realizó únicamente en enero y en los años de 1999 y 2000 sólo se realizaron los muestreos en febrero. Las colectas de quiste se realizaron principalmente en dos lotes salineros, a excepción de los dos últimos muestreos (1999 y 2000), en los cuales se realizó la colecta en varias charcas de la salina, que es donde se observó mayor cantidad de quiste. Cabe señalar que también se registró la presencia o ausencia de biomasa de Artemia. Los quistes se recogieron con palas de plástico y se transportaron en cubetas con salmuera hacia el laboratorio. La depuración del quiste consistió en eliminarles las basuras a través de lavados y filtrados con diferentes mallas $(500,300$ y $200 \mu \mathrm{m})$. Los quistes viables se separaron mediante el método de flotación y sedimentación (Castro y De LaraAndrade 1991); posteriormente se secaron en un horno para secado de alimento balanceado con extracción de aire seco a $38{ }^{\circ} \mathrm{C}$, por un período de $24 \mathrm{~h}$. El producto seco resultante se pesó (peso constante), se colocó en frascos color ámbar y se almacenó en un lugar fresco. Se evaluó la composición bioquímica (AOAC 1984) para determinar el valor nutricional (proteína, lípidos, fibra, ceniza y extracto libre de nitrógeno) de los quistes y nauplios de la población silvestre de Artemia de Real de Salinas y del lote comercial de A. franciscana.

Registro de parámetros ambientales: en cada colecta se registró la temperatura con un termómetro de mercurio $\left(0-50{ }^{\circ} \mathrm{C}\right)$ y la salinidad con un refractómetro ATAGO con rango entre 0-300 \% . Cuando la salinidad fue superior a los $300 \%$, se hicieron diluciones de la muestra de salmuera con agua destilada en proporción de 1:1 y se procedió a la determinación con el mismo instrumento. Los registros de precipitación pluvial se obtuvieron de la Comisión Nacional del Agua.

Calidad biológica de los quistes: la calidad biológica de los quistes se determinó utilizando las técnicas modificadas por Castro y Lara-Andrade (1991) y Gelabert et al. (1993), bajo las siguientes condiciones estándares de eclosión: 35 g/l de sal, aireación constante y 1000 lux de iluminación permanente. Cabe 
señalar que el equipo o instrumentos utilizados en las evaluaciones descritas a continuación, consistieron en una pipeta automática PIPETMAN P1000, un microscopio estereoscópico NOVA modelo ST623, una reglilla micrométrica, una cámara Bogorov, una balanza analítica OHAUS de $0.01 \mathrm{~g}$ de precisión y un microscopio óptico binocular Micromaster CK.

El \%H se determinó contando 100 quistes (diez repeticiones) con ayuda del microscopio estereoscópico, los cuales se colocaron en una caja petri con agua y junto a una fuente luminosa permanente durante $48 \mathrm{~h}$.

La HE se determinó incubando $1 \mathrm{~g}$ de quistes (tres repeticiones) en un recipiente cónico con capacidad de 11 durante $48 \mathrm{~h}$. Con la pipeta automática se tomaron 16 submuestras de $1 \mathrm{ml}$ a las 24 y $48 \mathrm{~h}$ y se contaron los nauplios. Se calcularon los promedios y se extrapolaron a 1 1. El porcentaje y eficiencia de eclosión, se determinaron con los quistes colectados en enero de 1998, debido a que fue una de los más antiguos y más abundantes, asumiendo que los resultados obtenidos podrían ser mejores con quistes de años posteriores.

La HR se determinó incubando $1 \mathrm{~g}$ de quistes (tres repeticiones) en un recipiente cónico de 11 de capacidad. Después de $5 \mathrm{~h}$ se realizaron muestreos cada hora, los cuales consistieron en tomar cinco submuestras de $1 \mathrm{ml}$ con la pipeta automática, colocarlos en cajas petri para observar con ayuda del microscopio estereoscópico la posible presencia de nauplios. Se determinó el tiempo en el que eclosionó el primer nauplio (To). En el momento en que se observó estabilidad en el número de organismos eclosionados, se consideró haber alcanzado el $100 \%$ de eclosión. Con esta información se establecieron los tiempos T10 y T90 que representan los tiempos en los que se alcanza el $10 \%$ y el $90 \%$ de eclosión. Estos datos permitieron conocer la sincronía de eclosión ( $\mathrm{Ts}=\mathrm{T} 90-\mathrm{T} 10)$.

La biomasa de eclosión (HO) se obtuvo incubando $1 \mathrm{~g}$ de quistes (tres repeticiones) en un recipiente de 11 de capacidad. Al cabo de $24 \mathrm{~h}$, los nauplios fueron cosechados y secados en un horno a $39{ }^{\circ} \mathrm{C}$ hasta peso constante, posteriormente éstos se pesaron en la balanza analítica.
Se evaluó el diámetro de los quistes y grosor del corion, los cuales se obtuvieron con la medición de 50 quistes hidratados y 50 descapsulados con la ayuda del microscopio óptico y la reglilla micrométrica. La diferencia de estas mediciones dividida entre dos, permitió conocer el grosor del corion (Gelabert et al. 1993). El procedimiento consistió en hidratar $200 \mathrm{mg}$ de quiste en un vaso de precipitado de $250 \mathrm{ml}$ con agua dulce durante $1 \mathrm{~h}$, hasta que se observaron totalmente esféricos. Para descapsular los quistes, éstos se hidrataron también por espacio de $1 \mathrm{~h}$. Posteriormente se descapsularon con hipoclorito de sodio y agua de mar en una proporción 1:1 (Castro, com. pers.).

Se determinó la longitud total de los nauplios, incubando $1 \mathrm{~g}$ de quiste durante $24 \mathrm{~h}$. Los nauplios recién eclosionados se capturaron y se fijaron con unas gotas de Solución de Davidson para inmovilizarlos. Se tomaron 50 organismos y se midieron utilizando el microscopio óptico y la reglilla.

\section{RESULTADOS}

El quiste de Artemia colectado en cada fecha de muestreo, la presencia o ausencia de biomasa observada, los parámetros fisicoquímicos y los registros de la precipitación pluvial se presentan en la cuadro 1. La composición bioquímica de los quistes y nauplios de ambas poblaciones se presentan en la cuadro 2 .

En la cuadro 3 se observa que el porcentaje de eclosión en el quiste capsulado de la población de Real de Salinas es mayor cuando se incrementa el período de incubación de $24 \mathrm{~h}$ $(2.05 \%)$ a 48 h (11.64 \%) y más aún cuando el quiste se descapsula (44.99 \% y $62.17 \%$ a las $24 \mathrm{~h} \mathrm{y} 48 \mathrm{~h}$, respectivamente), sin embargo comparativamente con la población de $A$. franciscana, las diferencias son notables en el quiste capsulado, no así en el quiste descapsulado. Con respecto a la eficiencia de eclosión, las diferencias entre ambas poblaciones son evidentes, superando en un $98.9 \%$ y $92.4 \%$ la eficiencia de eclosión la población de A. franciscana a la de Real de Salinas con 
CUADRO 1

Variables registradas en los muestreos realizados en las salinas de Real de Salinas, Calkini, Campeche

$\begin{array}{cccccc}\begin{array}{c}\text { Fecha } \\ (\mathrm{mes} / \mathrm{año})\end{array} & \begin{array}{c}\text { Quiste } \\ \text { colectado }(\mathrm{g})\end{array} & \begin{array}{c}\text { Biomasa de } \\ \text { Artemia }\end{array} & \begin{array}{c}\text { Salinidad }(\mathrm{g} / \mathrm{l}) \\ 01 / 97\end{array} & \begin{array}{c}\text { Temperatura } \\ \left({ }^{\circ} \mathrm{C}\right)\end{array} & \begin{array}{c}\text { Precipitación pluvial } \\ (\mathrm{mm})\end{array} \\ 02 / 97 & 500 & \text { Ausencia } & 32 & 46.4 \\ 03 / 97 & 0 & \text { Presencia } & 360 & 30 & 7.4 \\ 04 / 97 & 150 & \text { Ausencia } & 380 & 32 & 7.0 \\ 05 / 97 & 0 & \text { Presencia } & 340 & 32 & 13.0 \\ 06 / 97 & 0 & \text { Ausencia } & 270 & 32 & 15.0 \\ 07 / 97 & 0 & \text { Ausencia } & 190 & 32 & 90.0 \\ 09 / 97 & 0 & \text { Ausencia } & 80 & 35 & 156.9 \\ 10 / 97 & 0 & \text { Ausencia } & 50 & 33 & 222.1 \\ 11 / 97 & 1500 & \text { Ausencia } & 100 & 35 & 19.5 \\ 12 / 97 & 1800 & \text { Presencia } & 150 & 32.5 & 1.3 \\ 01 / 98 & 10700 & \text { Presencia } & 200 & 35 & 9.2 \\ 02 / 99 & 500 & \text { Presencia } & 270 & 32 & 48.2 \\ 02 / 00 & 900 & \text { Ausencia } & 270 & 33 & 0.0\end{array}$

*Comisión Nacional del Agua 1977-2000.

\section{CUADRO 2}

Valores en porcentaje de la composición bioquímica (base seca) de quistes y nauplios de las poblaciones de Artemia de Real de Salinas y A. franciscana

\begin{tabular}{|c|c|c|c|c|c|}
\hline Real de Salinas & Proteína & Lípidos & Fibra & Ceniza & $\begin{array}{l}\text { Extracto libre de } \\
\text { Nitrógeno }\end{array}$ \\
\hline \multicolumn{6}{|l|}{ Quiste } \\
\hline 1997 & 50.78 & 0.59 & 21.08 & 11.45 & 16.1 \\
\hline 1998 & 47.54 & 0.71 & 19.78 & 14.53 & 17.44 \\
\hline 1999 & 43.47 & 0.35 & 24.64 & 16.6 & 14.94 \\
\hline 2000 & 49.88 & 0.75 & 19.35 & 13.25 & 17.44 \\
\hline Nauplio & 50.5 & 9.50 & 15.0 & 14.0 & 11.0 \\
\hline \multicolumn{6}{|l|}{ A. franciscana } \\
\hline Quiste & 56.98 & 0.41 & 14.71 & 5.17 & 22.73 \\
\hline Nauplio & 58.20 & 19.27 & 3.69 & 15.24 & 3.60 \\
\hline
\end{tabular}

24 h y 48 h de incubación, respectivamente en quistes descapsulados.

El número de quistes por gramo, la tasa, sincronía y biomasa de eclosión de los quistes de las poblaciones en cuestión, se encuentran en la cuadro 4. La población de
Real de Salinas presentó un valor medio de $300900 \pm 7454$ quistes en comparación con el quiste de $A$. franciscana, con un promedio de $332667 \pm 21825$ quistes, encontrándose diferencias significativas $(\mathrm{p}<0.05)$ entre las dos poblaciones. 


\section{CUADRO 3}

Porcentaje (\%H) y eficiencia de eclosión (HE nauplios/gramo) de los quistes de las poblaciones de Artemia de Real de Salinas y A. franciscana

Artemia de Real de Salinas

Descapsulados

$\begin{array}{ccc}\text { Tiempo (h) } & 24 & 48 \\ \% \mathrm{H} & 44.99 & 62.17 \\ \mathrm{HE}(\mathrm{n} / \mathrm{g}) & 854 & 8042\end{array}$

Sin descapsular

$\begin{array}{cc}24 & 48 \\ 2.05 & 11.64\end{array}$

Artemia franciscana

\begin{tabular}{cccc}
\multicolumn{2}{c}{ Descapsulados } & \multicolumn{2}{c}{ Sin descapsular } \\
24 & 48 & 24 & 48 \\
57.22 & 56.53 & 54.55 & 54.70 \\
79208 & 106000 & - & -
\end{tabular}

\section{CUADRO 4}

Número de quistes por gramo, tasa (HR), sincronia (TS) y biomasa (HO) de eclosión, de la población de Artemia de Real de Salinas y A. franciscana

$\begin{array}{cccc}\text { Fúmero de } & \text { HR } & \text { TS } & \text { HO } \\ \text { quistes/gramo } & \text { (h) } & \text { (T90-T10) } & \text { (mg/g de quiste) }\end{array}$

$\begin{array}{ccccccc}\text { Real de Salinas } & & \text { T0 } & \text { T10 } & \text { T90 } & & \\ 1997 & 298966 & 18 & 33 & 61 & 28 & 34.0 \\ 1998 & 306857 & 18 & 36 & 61 & 25 & 56.67 \\ 1999 & 297103 & 19 & 32 & 58 & 26 & - \\ 2000 & 305010 & 20 & 35 & 55 & 20 & 56.68 \\ \text { A. franciscana } & 332667 & 14 & 19 & 45 & 26 & 370.00\end{array}$

El diámetro de los quistes hidratados capsulados y descapsulados, así como grosor de corion y longitud de nauplios se encuentran representados en la cuadro 5. Los valores correspondientes a 1997, representan el promedio durante el tiempo de observación señalado anteriormente, en los cuales se realizó colecta de quistes.

El diámetro de los quistes hidratados capsulados de $A$. franciscana $(230.21 \pm 12.49 \mu \mathrm{m})$

\section{CUADRO 5}

Comparación de la biometría de quistes y nauplios de la población de Artemia de Real de Salinas, Calkini, Campeche, México y Artemia franciscana, EEUU.

\begin{tabular}{lcccc}
\multicolumn{1}{c}{ Población (año) } & $\begin{array}{c}\text { Diámetro del quiste } \\
\text { hidratado capsulado } \\
(\mu \mathrm{m})\end{array}$ & $\begin{array}{c}\text { Diámetro del quiste } \\
\text { descapsulado } \\
(\mu \mathrm{m})\end{array}$ & $\begin{array}{c}\text { Grosor del corion } \\
(\mu \mathrm{m})\end{array}$ & $\begin{array}{c}\text { Longitud total } \\
\text { de nauplios } \\
(\mu \mathrm{m})\end{array}$ \\
$\begin{array}{l}\text { Real de Salinas } \\
(1997)\end{array}$ & $232.27 \pm 4.58$ & $222.5 \pm 13.6$ & $4.89 \pm 0.60$ & $391.70 \pm 2.33$ \\
$\begin{array}{l}\text { Real de Salinas } \\
(1998)\end{array}$ & $226.94 \pm 9.45$ & $219.46 \pm 12.42$ & $3.74 \pm 2.62$ & $383.23 \pm 12.42$ \\
$\begin{array}{l}\text { Real de Salinas } \\
(1999)\end{array}$ & $226.5 \pm 9.4$ & $218.7 \pm 12.3$ & $3.9 \pm 2.8$ & $385 \pm 12.0$ \\
$\begin{array}{l}\text { Real de Salinas } \\
(2000)\end{array}$ & $232.9 \pm 10.8$ & $225 \pm 12.9$ & $3.95 \pm 3.0$ & $385.3 \pm 12.9$ \\
$\begin{array}{l}\text { A. franciscana } \\
\text { (lote No. } 8131)\end{array}$ & $230.21 \pm 12.49$ & $216.96 \pm 13.71$ & $6.62 \pm 2.72$ & $424.70 \pm 30.08$ \\
$\begin{array}{l}\text { desviación estándar } \\
\text { fon }\end{array}$ & & &
\end{tabular}


fue muy similar al promedio $(230.5 \pm 4.14 \mu \mathrm{m})$ obtenido con la población de Real de Salinas. En cuanto a los quistes descapsulados de ésta última, son de mayor tamaño $(221.8 \pm 3.39 \mu \mathrm{m})$ que los de $A$. franciscana $216.96 \pm 13.71 \mu \mathrm{m}$ ). Con respecto al grosor del corion, éste es más pequeño $(4.38 \pm 0.68 \mu \mathrm{m})$ en la población de Real de Salinas que en la de $A$. franciscana $(6.62 \pm 2.72 \mu \mathrm{m})$. Sin embargo el análisis estadístico nos indicó que no existen diferencias significativas $(p>0.05)$ en estas variables entre ambas poblaciones.

El valor medio de la longitud de los nauplios de la población de Real de Salinas fue menor $(388.08 \pm 4.399 \mu \mathrm{m})$, comparativamente con la $A$. franciscana $(424.7 \pm 30.08 \mu \mathrm{m})$. Al respecto, la prueba $t$ de Student no reportó diferencias significativas $(\mathrm{p}<0.05)$ entre ambas poblaciones.

\section{DISCUSIÓN}

Batllori (1991) describe los cambios hidrológicos, físicos, químicos y biológicos en los sistemas acuáticos, debidos al paso de huracanes en la Península de Yucatán. Señala que estos fenómenos naturales traen como consecuencia cambios bruscos y recuperación lenta de los sistemas afectados. En este sentido, se considera que tal vez las secuelas del paso de los huracanes Opal y Roxana en septiembre de 1995, puedan ser una de las causas que marcaron las diferencias en la presencia de quiste y biomasa de Artemia en los muestreos. Además Lavens y Sorgeloos (2000) señalan que en un ecosistema natural, el clima y otros cambios ambientales interfieren en la producción de quiste y por lo tanto se presentan fluctuaciones impredecibles en la cosecha de quistes de un año a otro.

El número de quistes por gramo es un criterio de calidad que permite evaluar el peso del quiste y depende también de las condiciones fisicoquímicas del ambiente. El número de quistes por gramo promedio (300 900土7 454) de la población de Real de Salinas se encuentra entre los promedios informados por Gelabert
(1994) para la Artemia de Cuba colectada en los años de 1987, 1988 y 1989 (291 250, 311875 y 320000 quistes por gramo respectivamente). Castro y Gallardo (1993) informan para poblaciones de México, promedios de 369102 para Yavaros, Sonora, 380000 para Ceuta, Sinaloa, 358000 para San Luis Potosí y 377869 para la población de San Crisanto, Yucatán; siendo de éstas, geográficamente la más cercana a la población de Real de Salinas en el estado de Campeche.

Con respecto a las medidas de los quistes y de acuerdo a la revisión bibliográfica, el grosor del corion $(4.38 \mu \mathrm{m})$ de la población de Real de Salinas es pequeño en comparación con otras poblaciones. Los valores más cercanos a este resultado son los obtenidos por Enciso (1989), en la población de Ecatepec, estado de México $(5.05 \mu \mathrm{m})$, y la informada por Vanhaecke y Sorgeloos (1980) en la población del Gran Lago Salado de UTA $(5.45 \mu \mathrm{m})$. Castro (com. pers.), señala que el grosor del corion $(7.12 \mu \mathrm{m})$ para la población objeto de este estudio, es superior a lo que se encontró en este trabajo.

Alvarez y Sánchez (1994) señalan que las condiciones ambientales son las responsables fundamentales de las diferencias observadas en las características biométricas de los quistes. Con respecto al grosor del corion, Correa y Bückle (1993), mencionan que las diferencias también pueden ser causadas por las condiciones ambientales de su hábitat, confiriendo éstas, estrategias adaptativas para la supervivencia, donde los quistes de mayor tamaño, poseen mayores reservas de vitelo, lo que permite al nauplio contar con energía suficiente para romper el corion y por añadidura estos organismos tendrán mayor ventaja, cuando el alimento sea escaso en el medio.

Amat (1982) y Sorgeloos et al. (1986) mencionan que el corion de mayor grosor, se presenta como una respuesta para compensar la acción de la salinidad elevada y las altas temperaturas. Gelabert et al. (1993) mencionan que el corion tiene como principal función, proteger al embrión contra la radiación solar. El grosor del corion de los quistes de Real de Salinas fue menor con respecto a lo informado por 
otros autores, incluso para la misma población. Las condiciones de temperatura y salinidad (Cuadro 1) encontradas al momento de realizar los muestreos de quiste no parecen haber influido en el grosor del corion de la población objeto de este estudio.

En esta investigación se observó que al realizar el proceso de descapsulación, el corion $(4.38 \pm 0.68 \mu \mathrm{m})$ de la población de Real de Salinas se disolvió más lentamente, en comparación al corion de $A$. franciscana que fue más grueso $(6.62 \pm 3.0 \mu \mathrm{m})$. La resistencia observada del corion en la población de Real de Salinas al proceso de descapsulación, se puede decir que no se debió a su grosor. Se presume que otros atributos como la microestructura o composición química de las capas particulares de la cáscara, le confirieron mayor resistencia al corion de la población de Real de Salinas, los cuales de acuerdo a Anderson et al. (1970), influyen también en el grado de permeabilidad del corion.

Léger et al. (1986) señalan que el diámetro del quiste difiere ampliamente, de 224.7 a $284.9 \mu \mathrm{m}$ en quistes hidratados y de 207.3 a $266.3 \mu \mathrm{m}$ en quistes hidratados descapsulados. El quiste de la población de Real de Salinas capsulado $(230.5 \mu \mathrm{m})$ y descapsulado $(221.8 \mu \mathrm{m}) \mathrm{se}$ ubica dentro de estos rangos. Comparativamente con quistes de poblaciones cercanas a la región de San Crisanto, el diámetro del quiste capsulado es mayor a los de poblaciones como Celestún $(219.3 \mu \mathrm{m})$, Chuburná $(226.1 \mu \mathrm{m})$, Xtampú $(227.1 \mu \mathrm{m})$ y Coloradas $(221.4 \mu \mathrm{m})$, Yucatán (Torrentera-Blanco 1993). Sin embargo, el diámetro del quiste de la Artemia de Real de Salinas es pequeño en comparación con las poblaciones de Artemia del viejo mundo (Léger et al. 1986).

El porcentaje de eclosión en la población de Real de Salinas fue en general bajo y se incrementó en forma evidente con mayor tiempo de incubación y el tratamiento con la solución descapsulante; no así para $A$. franciscana, en la cual el tiempo de incubación y el proceso de descapsulación no marcaron diferencias. Es preciso recordar que el quiste que fue utilizado para determinar el porcentaje de eclosión y la eficiencia de eclosión fue de 1998. Si la antigüedad del quiste y la calidad de almacenamiento influyeron en estos resultados, se presume que éstos pudieron ser mejores con quistes de años posteriores.

Torrentera-Blanco et al. (1993) señalan para la población de Celestún, Yucatán, un incremento en el porcentaje de eclosión cuando descapsuló el quiste e incubó durante $72 \mathrm{~h}$. Además mencionan que la estrategia de esta población, fue la de eclosionar pocos huevos en condiciones intermedias de eclosión en laboratorio, con salinidad de 35-60 g/l y temperaturas de $25-30{ }^{\circ} \mathrm{C}$, lo que indica un estado latente muy largo, sugiriendo que esta población tiene un banco de huevos durmiente, similar al de otros crustáceos en hábitats temporales. Dada la cercanía entre Celestún y Real de Salinas y la inexistencia de barreras geográficas, se puede inferir que se trata de la misma población, por lo que la baja tasa de eclosión que presentó el quiste de la población de Real de Salinas, con las condiciones estándar de eclosión se pudo deber al estado de latencia señalado por Torrentera-Blanco et al. (1993). Al respecto, estos investigadores señalan que la fuerte diapausa de los quistes está inducida por los procesos de hidratación y deshidratación a los que están sometidos en ambientes naturales. En este sentido, dichos procesos provocan que parte de las reservas energéticas de los quistes, se pierdan en intentos fallidos de eclosión.

Aunado a lo anterior, el pobre porcentaje de eclosión de los quistes, se puede deber también a las diferencias genéticas entre las poblaciones, factores climáticos y físicoquímicos del ambiente, técnicas de limpieza y procesado del quiste (Sorgeloos et al. 1986, TorrenteraBlanco et al.1993).

La salinidad interfiere en los niveles de glicerol que los quistes necesitan para incrementar la presión osmótica intra quística y promover la eclosión. Como resultado, la óptima salinidad del agua para la eclosión de los quistes, varía de una población a otra (Lavens y Sorgeloos 1987).

Con base en la escasa información relacionada con el estudio de la Artemia de Real 
de Salinas, se consideró conveniente manejar condiciones estándares de eclosión en este trabajo, dado que se trata de un primer acercamiento para conocer los requerimientos de esta población, sin embargo esto no significa que hayan sido las mejores condiciones para obtener su máximo rendimiento. Las experiencias de otros autores, los resultados obtenidos en el presente trabajo y la importancia que representa el porcentaje de eclosión en acuicultura (en los centros de larvicultura se prefieren lotes de quistes que presenten el $80 \%$ de eclosión) (Amat 1980, Hontoria et al. 1989), nos indican que es necesario evaluar valores diferentes a los considerados como estándar de eclosión y diferentes rangos de todas aquellas variables que influyen en la calidad biológica del quiste, con el fin de conocer cuales son las condiciones óptimas para obtener los máximos rendimientos, los cuales contribuirán a determinar su viabilidad de explotación para beneficio de la actividad acuícola de la región.

Sin embargo, también hay que considerar que los quistes de Artemia descapsulados (también llamados quistes libres de cáscara) han sido utilizados como alimento directo para peces y decápodos, los cuales presentan como principales ventajas su tamaño pequeño y su contenido energético superior al de un nauplio (Léger et al. 1986). La principal desventaja es su sedimentación, la cual puede ser contrarrestada utilizando los quistes descapsulados secos, los cuales se han utilizado como alimento inicial para larvas de camarón (Lavens y Sorgeloos 1998). Abreu-Grobois y Peralta-Caballero (1991) señalan que los quistes descapsulados promueven su crecimiento a niveles equivalentes a los obtenidos con nauplios. Actualmente se sigue evaluado la incorporación de los quistes descapsulados en las dietas para peces (Weirich et al. 2000). En el caso de los quistes con poca calidad de eclosión, se mencionan como una buena opción para utilizarlos en este sentido (Lavens y Sorgeloos 1998). Este aspecto es sumamente importante, dadas las características que presentó el quiste de Real de Salinas.
Léger et al. (1986) señala que de acuerdo al origen de la población, las longitudes de los nauplios instar I se ubican en el rango de 428 a $517 \mu \mathrm{m}$. Los nauplios de Real de Salinas son pequeños $(388.08 \mu \mathrm{m})$, siendo menores también a los de $A$. franciscana $(424.7 \mu \mathrm{m})$ comercial y los informados por TorrenteraBlanco (1993) para las poblaciones de Celestún

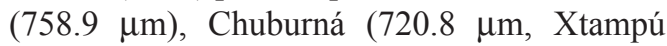
$(556.1 \mu \mathrm{m})$ y Coloradas $(458.5 \mu \mathrm{m})$, Yucatán; regiones cercanas a la zona de estudio.

Los valores más cercanos a la longitud total de los nauplios de la población de Real de Salinas $(388.08 \mu \mathrm{m})$, son los informados por Castro et al. (en prep) $(411.01 \mu \mathrm{m})$ para la misma población, Ceuta, Sinaloa $(386 \mu \mathrm{m})$ y Yavaros Sonora (408 $\mu \mathrm{m})$, México (Castro y Gallardo 1993) y Guantánamo, Cuba (406 $\mu \mathrm{m})$ (Gelabert et al. 1993). El tamaño pequeño del nauplio de la población de Real de Salinas es una cualidad de gran importancia, ya que las larvas de peces y crustáceos, requieren en su alimentación presas de alto valor nutritivo y de talla pequeña.

Léger et al. (1986), señalan que hay una considerable variación en la composición bioquímica de Artemia. Las causas principales de dichas variaciones, son principalmente los diferentes métodos de extracción y análisis, las diferentes etapas de desarrollo de Artemia y diferencias atribuidas a la ubicación geográfica de la población.

La composición bioquímica de los quistes de ambas poblaciones, presentó la diferencia más amplia en el contenido de ceniza; esto se atribuye a que por un lado se utilizó quiste de una marca comercial, cuyo proceso de limpieza y secado es diferente al que se le proporcionó al quiste de Real de Salinas. El mencionado manejo, se considera que también influyó para que se presentaran notorias diferencias en la biomasa de eclosión (HO) entre ambas poblaciones.

Las diferencias nutritivas en los quistes de Artemia silvestres dependen de los caprichos de la naturaleza, proveyendo a los acuicultores quiste de calidad variable en función del tiempo (Léger et al. 1986). Bengtson (1991) 
señala que la composición bioquímica de los nauplios de Artemia varia ampliamente y que los valores se encuentran entre 37-71 \% para proteínas, con un promedio excluyendo los extremos de $50 \%$ (Léger et al. 1986), de 12 a $30 \%$ para lípidos y de 4-21\% para cenizas. El valor proteico $(50.5 \%)$ de los nauplios de la población de Real de Salinas, se ubicó dentro del promedio señalado por Léger et al. (1986). Las cenizas $(14.0 \%)$ se encuentran dentro del rango señalado por Bengtson (1991); no así los lípidos $(9.50 \%)$. La variación, se atribuye principalmente a que se trata de una población diferente a las informadas en la literatura.

Se considera conveniente hacer análisis específicos de aminoácidos y ácidos grasos para la población de Artemia en cuestión y/o evaluar su calidad a través de su uso como alimento en el crecimiento, sobrevivencia, etc. de un organismo en particular. Ambas evaluaciones son estrategias que pueden ser utilizadas para demostrar su efectividad como alimento en acuicultura. Sin embargo también es necesario tener en mente que el valor especial de Artemia como fuente de alimento no es debida tanto a su composición nutritiva, sino al gran alcance para su adecuada producción, su óptima disponibilidad física como una presa móvil de tamaño adecuado y las bondades que presenta para la bioencapsulación de componentes vitales, convirtiéndolo de un alimento deficiente a una dieta supranatural (Léger et al. 1986).

El abastecimiento de quistes provenientes del medio natural es incierto, dado los factores que influyen en su producción; en este sentido, es necesario disponer de información sobre la ecología de los hábitats de Artemia. Esto significa que no hay una provisión estable de quistes que pueda ser garantizada; pero la diversificación de las fuentes, va limitar el riesgo de desabastecimiento súbito debido a los cambios ambientales (Lavens y Sorgeloos 1998). Los resultados del presente trabajo a pesar de ser preliminares, son alentadores; sin embargo, se requieren más evaluaciones para conocer la potencialidad de las charcas salineras en esa región del sureste de México, el cual si bien es cierto no contribuiría significativamente en el abasto de Artemia en el ámbito nacional, si pudiese contribuir a pequeña escala al abasto local del producto, ahora que el desarrollo de la acuicultura en la región esta cobrando día a día más importancia.

\section{AGRADECIMIENTOS}

Nuestro más sentido y amplio agradecimiento al Gobierno del Estado de Campeche y a la Comisión Nacional para el Conocimiento y Uso de la Biodiversidad (CONABIO) por el apoyo financiero para llevar a cabo esta investigación y al laboratorio de Ecología y Biología Marina Experimental, de la Universidad Nacional Autónoma de México, en Ciudad del Carmen, Campeche, por habernos facilitado sus instalaciones y brindado apoyo técnico y logístico.

\section{RESUMEN}

Se utilizaron quistes de Artemia recolectados en períodos de febrero de 1997 a febrero del 2000 en las salinas de Real de Salinas, Campeche, México y como especie de comparación Artemia franciscana (lote No. 8131 Microfeast Artemia Cysts, Texas, EEUU.). Se determinó el número de quistes por gramo, porcentaje de eclosión $(\% \mathrm{H})$, eficiencia, tasa, sincronía y biomasa de eclosión, así como diámetro de quistes y longitud de los nauplios (instar I) de ambas poblaciones. Para la población de Real de Salinas, el diámetro de quistes capsulados y descapsulados en promedio fue de $230.5 \pm 4.14$ y $221.8 \pm 3.39 \mu \mathrm{m}$, respectivamente. El grosor del corion de $4.35 \pm 0.68 \mu \mathrm{m}$ y la longitud del nauplio de $388.11 \pm 4.39 \mu \mathrm{m}$, siendo este último de los más pequeños que se hayan publicado en la literatura. Para la población de $A$. franciscana comercial, el diámetro del quiste capsulado y descapsulado presentó valores promedio de $230.21 \pm 12.49$ y $216.96 \pm 13.71 \mu \mathrm{m}$, respectivamente. Respecto al grosor del corion y longitud del nauplio, los valores fueron de $6.62 \pm 2.72$ y $424.70 \pm 30.08 \mu \mathrm{m}$, respectivamente. El valor proteico de los quistes (47.91\%) y nauplios (50.5\%) de Artemia de la población de Real de Salinas, se considera adecuado para uso como alimento en acuicultura. La población de Real de Salinas presenta bondades para su aprovechamiento en la acuicultura regional.

Palabras clave: artemia, alimento vivo, crustáceo, nutrición, quiste. 


\section{REFERENCIAS}

Abreu-Grobois, F.A. \& M.E. Peralta-Caballero. 1991. A comparison of the use of decapsulated Artemia cysts and nauplii as food for Penaeus vannamei larvae, p. 128-131. In P. Lavens, P. Sorgeloos, E. Jaspers \& F. Ollevier (eds.). Fish and Crusta cean Larviculture Symposium. European Aquaculture Society, Gent, Bélgica.

Alvarez, Z. \& R. Sánchez. 1994. Actas del VIII Congreso Latinoamericano de Acuicultura de Santa Fe de Bogotá. Variaciones en la calidad de la eclosión de la cepa de Artemia Las Cumaraguas-Paraguana, Venezuela. Congreso Latinoamericano de Acuicultura, Bogotá, Colombia. p. 562.

Amat, F. 1980. Antecedentes, estado actual y perspectivas del empleo de Artemia salina en acuicultura. In Informe Técnico presentado a Instituto de Investigaciones Pesqueras. Consejos Superiores de Investigaciones Científicas, Barcelona, España.

Amat, F. 1982. Diferenciación y distribución de las poblaciones de Artemia de España. IV. Biometría de quistes y nauplios. Investig. Pesq. 46: 55-62.

Anderson, E.D., J.H. Lochhed, M.S. Lochhead \& E. Huebner. 1970. The origin and structure of the tertiary envelope in thick-shell eggs of the brine shrimp, Artemia. J. Ultrastruct. Res. 32: 497-525.

AOAC (Association of Official Analytical Chemists). 1984. Official methods of analysis. Association of Official Analytical Chemists, Washington, D.C., EEUU.

Batllori, S.E. 1991. Caracterización ecológica del refugio faunístico "Ría de Celestún" al norte de la Península de Yucatán. Pueblo de pescadores en áreas marinas y de campesinos-pescadores en áreas palustres y estuarinas. CINVESTAV-IPN-Unidad Mérida, Yucatán, México. 83 p.

Bengtson, D.A., P. Leger \& P. Sorgeloos. 1991. Use of Artemia as a food source for aquaculture, p. 255-285. In R.A. Browne, P. Sorgeloos \& C.N.A.Trotman (eds.). Artemia Biology. CRC, Boca Raton, Florida, EEUU.

Castro, M.J. \& R. De Lara-Andrade. 1991. Técnicas para el manejo de quistes de Artemia sp. Universidad Autónoma Metropolitana-Xochimilco, México, DF., México. 47 p.

Castro, T. \& C. Gallardo. 1993. Artemia sp. División de ciencias biológicas y de la salud. Departamento el Hombre y su Ambiente. Universidad Autónoma Metropolitana Xochimilco, México, DF., México. 43 p.
Comisión Nacional del Agua. 1996. Plano Topográfico de los lotes salineros de Real de Salinas, Calkiní. Departamento de Aguas Superficiales, Campeche, México.

Comisión Nacional del Agua. 1977-2000. Estadística a tiempo real de precipitación pluvial, estación Isla Arena, Calkiní. Gerencia Estatal de Campeche, Campeche, México.

Correa, S.F. \& R.L.F. Bückle. 1993. Morfología y biometría de cinco poblaciones de Artemia franciscana (Anostraca: Artemiidae). Rev. Biol. Trop. 41:103-110.

Diario Oficial. 1979. Decreto del área conocida como Ría Celestún como zona de refugio faunístico entre los municipios de Celestún y Calkiní, en los Estados de Yucatán y Campeche, el 19 de julio de 1979, Gobierno de México, México, DF., México. 2 p.

Diario Oficial. 1996. Caracterización ecológica ambiental y de los recursos naturales de la región de los petenes en Campeche, Campeche. Gobierno Constitucional del Estado de Campeche, Campeche, México. 426 p

Enciso, P. 1989. Comparación de algunas características de dos poblaciones de Artemia sp. de San Francisco, Cal. USA y Ecatepec, Estado de México. Tesis de Licenciatura, Universidad Autónoma Metropolitana, Xochimilco, México, DF., México. 92 p.

Gelabert, R.F. 1994. Artemia. Ensayos de caracterización y su empleo. Universidad de la Habana, Habana, Cuba. 15 p.

Gelabert, R., R. Sánchez \& L. Solís. 1993. Valoración de la calidad de una cepa cubana de Artemia. Rev. Invest. Marinas, Habana, Cuba 14: 92-101.

Holt, G.U. \& F. Sun. 1991. Lipase activity and total lipid content during early development of red drum Sciaenops acellatus, p. 30-33. In P. Lavens, P. Lavens, P. Sorgeloos, E. Jaspers \& F. Oliver (eds.). Larvi '91. Fish and Crustacean Larviculture Symposium. Gent, Bélgica.

Hontoria, F., J. Navarro, I. Varo \& F. Amat. 1989. Utilization of Artemia cyst in marine larvae cultures: a model of quality evaluation. Aquacult. Eng. 8: 127-138.

Lavens, P. \& P. Sorgeloos 1987. The cryptobiotic state of Artemia cyst, its diapause deactivation and hatching: a review, p. 27-62. In P. Sorgeloos, D.A. Bengtson, W. Decleir \& E. Jaspers (eds.). Artemia Res. \& Applications. Universa, Wetteren, Bélgica.

Lavens, P. \& P. Sorgeloos. 1998. Proceedings Aquaculture Brazil '98 Conference. Present status and prospects 
of the use of Artemia cysts and biomass in shrimp farming. Recife, Brasil. 28 p.

Lavens, P. \& P. Sorgeloos. 2000. The history, present status and prospects of the availability of Artemia cyst for aquaculture. Aquaculture 181: 397-403.

Léger, P., D.A. Bengtson, K.L. Simpson \& P. Sorgeloos. 1986. The use and nutritional value of Artemia as a food source. Oceanogr. Mar. Biol. Ann. Rev. 24: 521-623.

Sorgeloos, P., P. Lavens, P. Léger, W. Tackaert \& D. Versichele. 1986. Manual para el cultivo y uso de Artemia en acuicultura. FAO, Roma, Italia. 301 p.
Torrentera-Blanco, L. 1993. Ecology and evolution of Yucatan Peninsula. Artemia. Doctor Thesis, University of Wisconsin, Wisconsin, EEUU. 104 p.

Vanhaecke, P. \& P. Sorgeloos. 1980. The biometrics of Artemia strains from different geographical origin, p. 393-405. In G. Persoone, P. Sorgeloos, O. Roels \& E. Jaspers (eds.). International study on Artemia . Universal, Wetteren, Bélgica.

Weirich, Ch.R., R.C. Reigh \& D.W.III. Glenn. 2000. Evaluation of decapsulated Artemia cysts in hatchery diets for channel catfish Ictalurus punctatus fry and effects on subsequent fingerling production. J. World Aquacult. Soc. 31: 609-617. 
